\title{
NONEXPANSIVE MAPPINGS AND EXPANSIVE MAPPINGS ON THE UNIT SPHERES OF SOME $F$-SPACES
}

\author{
DONG-NI TAN
}

\author{
(Received 9 July 2009)
}

\begin{abstract}
This paper gives a characterization of nonexpansive mappings from the unit sphere of $\ell^{\beta}(\Gamma)$ onto the unit sphere of $\ell^{\beta}(\Delta)$ where $0<\beta \leq 1$. By this result, we prove that such mappings are in fact isometries and give an affirmative answer to Tingley's problem in $\ell^{\beta}(\Gamma)$ spaces. We also show that the same result holds for expansive mappings between unit spheres of $\ell^{\beta}(\Gamma)$ spaces without the surjectivity assumption.
\end{abstract}

2000 Mathematics subject classification: primary 46A16; secondary 46B20.

Keywords and phrases: nonexpansive mapping, expansive mapping, isometric extension, Tingley's problem, $\ell^{\beta}(\Gamma)$ spaces.

\section{Introduction}

A mapping $V$ between two metric spaces $\left(X, d_{X}\right)$ and $\left(Y, d_{Y}\right)$ is called nonexpansive if it is a 1-Lipschitz map. That is,

$$
d_{Y}(V(x), V(y)) \leq d_{X}(x, y) \quad \forall x, y \in X .
$$

The mapping $V$ is called an isometry if equality holds in (1.1) for all $x, y \in X$, and it is called expansive if ' $\leq$ ' is replaced by the inverse inequality ' $\geq$ '.

By a direct compactness argument or by Freudenthal and Hurewicz's result [9], every nonexpansive map from a compact metric space onto itself must be an isometry. This does not always hold with the assumption of compactness replaced by boundedness in infinite-dimensional metric linear spaces. For example, a map $T: B\left(\ell^{p}\right) \rightarrow B\left(\ell^{p}\right)$ defined by $T\left(\xi_{1}, \xi_{2}, \xi_{3}, \ldots, \xi_{n}, \ldots\right)=\left(\xi_{2}, \xi_{3}, \ldots, \xi_{n}, \ldots\right)$ for all $\left\{\xi_{n}\right\}_{n \geq 1}$ in $B\left(\ell^{p}\right)$ where $B\left(\ell^{p}\right)$ denotes the unit ball of $\ell^{p}$ and $0<p \leq \infty$ is such a nonexpansive but not isometric map from $B\left(\ell^{p}\right)$ onto itself. However, what interests us is such maps defined only on the unit sphere, which can be connected with the isometric extension problem raised by Tingley in [12] and described as follows.

This work was partially supported by Research Foundation for Doctor Programme (20060055010) and National Natural Science Foundation of China (10871101).

(C) 2010 Australian Mathematical Publishing Association Inc. 0004-9727/2010 \$16.00 
Let $E$ and $F$ be normed spaces with unit spheres $S(E)$ and $S(F)$, respectively. Suppose that $V_{0}: S(E) \rightarrow S(F)$ is an onto isometry. Is there a linear isometry $V: E \rightarrow F$ such that $\left.V\right|_{S(E)}=V_{0}$ ?

In recent years, Ding and his students have been working on this topic and have obtained many important results (see $[1-7,10,13,15]$ ).

Ding [2] showed that every onto nonexpansive map between unit spheres of Hilbert spaces is an isometry and answered Tingley's problem affirmatively for Hilbert spaces. In recent work [11], the author proved that the only nonexpansive mappings from the unit sphere of $\mathcal{L}^{\infty}(\Gamma)$-type spaces (including $c_{00}, c, \ell^{\infty}$ ) onto the unit sphere of $\mathcal{L}^{\infty}(\Delta)$ are those arising from a bijection between $\Delta$ and $\Gamma$ and a sign pattern. This result yields the fact that such maps are isometries and an affirmative answer to Tingley's problem for $\mathcal{L}^{\infty}(\Gamma)$-type spaces. A similar result for $\ell^{p}(\Gamma)$ spaces where $1<p<\infty$ can be obtained by combining the main result in [3] with that of [8]. For the case $p=1$, Wang [14] established that every expansive map $T$ from $S\left(\ell^{1}(\Gamma)\right)$ onto $S\left(\ell^{1}(\Delta)\right)$ with an additional condition $\bigcup_{\gamma \in \Gamma} \operatorname{supp} T\left(e_{\gamma}\right)=\Delta$ is an isometry and can be linearly and isometrically extended to $\ell^{1}(\Gamma)$. In this paper, we extend these results to $F$-spaces $\ell^{\beta}(\Gamma)$ where $0<\beta \leq 1$, and in the $\ell^{1}(\Gamma)$ case we point out that the condition $\bigcup_{\gamma \in \Gamma} \operatorname{supp} T\left(e_{\gamma}\right)=\Delta$ in [14] can be removed.

Throughout this paper, we consider spaces over the real field. Given a nonempty index set $\Gamma$, for every $0<\beta \leq 1$, the space

$$
\ell^{\beta}(\Gamma)=\left\{x=\left\{\xi_{\gamma}\right\}_{\gamma \in \Gamma}: \sum_{\gamma \in \Gamma}\left|\xi_{\gamma}\right|^{\beta}<\infty\right\}
$$

is known as an $F$-space with an $F$-norm $\|x\|=\sum\left|\xi_{\gamma}\right|^{\beta}$. As usual, for every $x=$ $\left\{\xi_{\gamma}\right\}_{\gamma \in \Gamma} \in \ell^{\beta}(\Gamma)$, supp $x=\left\{\gamma \in \Gamma: \xi_{\gamma} \neq 0\right\}$ and $S\left(\ell^{\beta}(\Gamma)\right)$ denotes the unit sphere of $\ell^{\beta}(\Gamma)$.

\section{Main results}

Lemma 2.1. Let $x, y \in \ell^{\beta}(\Gamma)$. Then

$$
\|x+y\|=\|x\|+\|y\|
$$

if and only if supp $x \cap \operatorname{supp} y=\emptyset$ for $0<\beta<1$ and $x \cdot y \geq 0$ for $\beta=1$, where $x \cdot y \geq 0$ means $x(\gamma) \cdot y(\gamma) \geq 0$ for every $\gamma \in \Gamma$.

PROOF. The proof in the case of $\beta=1$ is trivial. For $0<\beta<1$, observe that the function $f(t)=t^{\beta}$ is strictly concave on $(0, \infty)$. It follows that

$$
|\xi+\eta|^{\beta} \leq|\xi|^{\beta}+|\eta|^{\beta}
$$

for all $\xi, \eta \in \mathbb{R}$ and equality holds if and only if $\xi \cdot \eta=0$. The desired result is easily obtained from this. 
LEMMA 2.2. Let $x \in S\left(\ell^{\beta}(\Gamma)\right)$. Then for every $\gamma \in \Gamma$,

$$
\max \left\{\left\|x+e_{\gamma}\right\|,\left\|x-e_{\gamma}\right\|\right\} \geq 2^{\beta} .
$$

PROOF. As $\|x\|=1$, it is easy to see that

$$
\max \left\{\left\|x+e_{\gamma}\right\|,\left\|x-e_{\gamma}\right\|\right\}=(|x(\gamma)|+1)^{\beta}+1-|x(\gamma)|^{\beta} .
$$

Since the function $\varphi(t)=(1+t)^{\beta}-t^{\beta}$ is decreasing on $[0, \infty)$, it follows that

$$
(|x(\gamma)|+1)^{\beta}+1-|x(\gamma)|^{\beta}=1+\varphi(|x(\gamma)|) \geq 1+\varphi(1)=2^{\beta},
$$

which completes the proof.

LEMMA 2.3. Let $T: S\left(\ell^{\beta}(\Gamma)\right) \rightarrow S\left(\ell^{\beta}(\Delta)\right)$ be a nonexpansive map. For each $\delta \in \Delta$, if $\pm e_{\delta} \in T\left(S\left(\ell^{\beta}(\Gamma)\right)\right)$, then there is a unique $\gamma \in \Gamma$ and a sign $\theta_{\delta}$ such that

$$
T\left( \pm e_{\gamma}\right)= \pm \theta_{\delta} e_{\delta}
$$

PRoOF. The hypothesis $\pm e_{\delta} \in T\left(S\left(\ell^{\beta}(\Gamma)\right)\right)$ ensures that there exist $x, y \in S\left(\ell^{\beta}(\Gamma)\right)$ such that $T(x)=e_{\delta}$ and $T(y)=-e_{\delta}$. We first claim that $x$ and $y$ are dependent, that is,

$$
x=-y \text {. }
$$

Assume that the claim is not true. Define a map $f:[0,1] \rightarrow S\left(\ell^{\beta}(\Gamma)\right)$ by

$$
f(\lambda)=\frac{(1-\lambda) x+\lambda y}{\|(1-\lambda) x+\lambda y\|^{1 / \beta}} .
$$

It is clear that $\{f(\lambda): \lambda \in[0,1]\}$ is a connected path from $x$ to $y$. Hence the map

$$
\phi(\lambda)=\left\|T(f(\lambda))+e_{\delta}\right\|-\left\|T(f(\lambda))-e_{\delta}\right\|
$$

is continuous on $[0,1]$. Since $\phi(0)=2^{\beta}$ and $\phi(1)=-2^{\beta}$, we can find $\lambda_{0} \in(0,1)$ such that $\phi\left(\lambda_{0}\right)=0$, that is,

$$
\left\|T\left(f\left(\lambda_{0}\right)\right)+e_{\delta}\right\|=\left\|T\left(f\left(\lambda_{0}\right)\right)-e_{\delta}\right\| .
$$

The definition of the norm in $\ell^{\beta}(\Delta)$ yields $T\left(f\left(\lambda_{0}\right)\right)(\delta)=0$, and thus

$$
\left\|T\left(f\left(\lambda_{0}\right)\right)+e_{\delta}\right\|=\left\|T\left(f\left(\lambda_{0}\right)\right)-e_{\delta}\right\|=2 .
$$

This shows that

$$
\left\|f\left(\lambda_{0}\right)-y\right\|=\left\|f\left(\lambda_{0}\right)-x\right\|=2 .
$$

By Lemma 2.1 we get that for $0<\beta<1$, supp $f\left(\lambda_{0}\right) \cap(\operatorname{supp} x \cup \operatorname{supp} y)=\emptyset$ and for $\beta=1, f\left(\lambda_{0}\right) \cdot x \leq 0$ and $f\left(\lambda_{0}\right) \cdot y \leq 0$. This is impossible by the definition of $f$. Therefore the claim is proved. 
We next show that supp $x$ is a singleton. If this does not hold, then there is a $\gamma_{1} \in \Gamma$ satisfying $0<\left|x\left(\gamma_{1}\right)\right|<1$. Write $x_{1}=x-2 x\left(\gamma_{1}\right) e_{\gamma_{1}}$. Then by the claim

$$
\begin{aligned}
\left\|T\left(x_{1}\right)-e_{\delta}\right\| & =\left\|T\left(x_{1}\right)-T(x)\right\| \leq\left\|x_{1}-x\right\|=2^{\beta}\left|x\left(\gamma_{1}\right)\right|^{\beta}<2^{\beta}, \\
\left\|T\left(x_{1}\right)+e_{\delta}\right\| & =\left\|T\left(x_{1}\right)-T(-x)\right\| \leq\left\|x_{1}+x\right\|=2^{\beta}\left(1-\left|x\left(\gamma_{1}\right)\right|^{\beta}\right)<2^{\beta} .
\end{aligned}
$$

This contradicts Lemma 2.2 and therefore supp $x$ is a singleton.

Let $\{\gamma\}=\operatorname{supp} x$ and $\theta_{\delta}=x(\gamma)$. Noticing that the uniqueness of $\gamma$ is easily obtained from the claim, this completes the proof.

We are now ready to present one of our main results.

THEOREM 2.4. Let $T: S\left(\ell^{\beta}(\Gamma)\right) \rightarrow S\left(\ell^{\beta}(\Delta)\right)$ be a surjective nonexpansive map. Then $T$ is an isometry and there is a family of signs $\left\{\theta_{\delta}\right\}_{\delta \in \Delta}$ and a bijection $\sigma: \Delta \rightarrow \Gamma$ such that, for any element $x \in S\left(\ell^{\beta}(\Gamma)\right)$,

$$
T(x)(\delta)=\theta_{\delta} x(\sigma(\delta)) \quad \forall \delta \in \Delta .
$$

PROOF. It is evident that $T$ is an isometry if there is a family of signs $\left\{\theta_{\delta}\right\}_{\delta \in \Delta}$ and a bijection $\sigma: \Delta \rightarrow \Gamma$ such that (2.1) holds. Thus it suffices to prove this. By Lemma 2.3 we can define $\sigma: \Delta \rightarrow \Gamma$ and $\left\{\theta_{\delta}\right\}_{\delta \in \Delta}$ such that

$$
T\left( \pm e_{\sigma(\delta)}\right)= \pm \theta_{\delta} e_{\delta} \quad \forall \delta \in \Delta .
$$

It is obvious that $\sigma$ is injective. To see that $\sigma$ is surjective and that (2.1) holds, for every $y=\sum \eta_{\delta} e_{\delta} \in S\left(\ell^{\beta}(\Delta)\right)$, take $x=\sum \xi_{\gamma} e_{\gamma} \in S\left(\ell^{\beta}(\Gamma)\right)$ such that $T(x)=y$.

For any $\delta \in \Delta$ with $\xi_{\sigma(\delta)} \neq 0$,

$$
\left\|y-\operatorname{sign}\left(\xi_{\sigma(\delta)}\right) \theta_{\delta} e_{\delta}\right\|=\left|\eta_{\delta}-\operatorname{sign}\left(\xi_{\sigma(\delta)}\right) \theta_{\delta}\right|^{\beta}+1-\left|\eta_{\delta}\right|^{\beta} .
$$

On the other hand, clearly,

$$
\left\|x-\operatorname{sign}\left(\xi_{\sigma(\delta)}\right) e_{\sigma(\delta)}\right\|=\left(1-\left|\xi_{\sigma(\delta)}\right|\right)^{\beta}+1-\left|\xi_{\sigma(\delta)}\right|^{\beta} .
$$

The fact that $T$ is nonexpansive and (2.2) then give

$$
\begin{aligned}
\left(1-\left|\eta_{\delta}\right|\right)^{\beta}-\left|\eta_{\delta}\right|^{\beta} & \leq\left|\eta_{\delta}-\operatorname{sign}\left(\xi_{\sigma(\delta)}\right) \theta_{\delta}\right|^{\beta}-\left|\eta_{\delta}\right|^{\beta} \\
& \leq\left(1-\left|\xi_{\sigma(\delta)}\right|\right)^{\beta}-\left|\xi_{\sigma(\delta)}\right|^{\beta} .
\end{aligned}
$$

Noticing that $\phi(t)=(1-t)^{\beta}-t^{\beta}$ is decreasing on $[0,1]$, we see that

$$
\left|\eta_{\delta}\right| \geq\left|\xi_{\sigma(\delta)}\right|
$$

Thus if supp $x \subset \sigma(\Delta)$, then by (2.4),

$$
1=\sum_{\delta \in \Delta}\left|\xi_{\sigma(\delta)}\right| \leq \sum_{\delta \in \Delta}\left|\eta_{\delta}\right|=1 .
$$


As a result,

$$
\left|\eta_{\delta}\right|=\left|\xi_{\sigma(\delta)}\right|
$$

and inequality (2.3) turning out to be an equality obviously implies that

$$
\operatorname{sign}\left(\eta_{\delta}\right)=\operatorname{sign}\left(\xi_{\sigma(\delta)}\right) \theta_{\delta} .
$$

Since Equations (2.5) and (2.6) have already established that (2.1) holds for all $x \in S\left(\ell^{\beta}(\Gamma)\right)$ satisfying supp $x \subset \sigma(\Delta)$, to finish the proof we only need to show that $\sigma$ is surjective. Suppose to the contrary that there is a $\gamma_{0} \in \Gamma \backslash \sigma(\Delta)$. Choose $\delta_{0} \in \operatorname{supp} T\left(e_{\gamma_{0}}\right)$ and put

$$
x_{0}^{ \pm}=\frac{1}{2^{1 / \beta}} e_{\gamma_{0}} \pm \frac{1}{2^{1 / \beta}} e_{\sigma\left(\delta_{0}\right)} \quad \text { and } \quad \eta_{\delta_{0}}^{ \pm}=T\left(x_{0}^{ \pm}\right)\left(\delta_{0}\right) .
$$

It is easy to see from Lemma 2.3 that supp $T\left(x_{0}^{+}\right)$cannot be a singleton. Thus we can let $\delta_{1} \in \operatorname{supp} T\left(x_{0}^{+}\right)$satisfy $\delta_{1} \neq \delta_{0}$. Then write

$$
\eta_{\delta_{1}}=T\left(x_{0}^{+}\right)\left(\delta_{1}\right) \quad \text { and } \quad x_{1}=\frac{1}{2^{1 / \beta}} e_{\sigma\left(\delta_{0}\right)}-\frac{1}{2^{1 / \beta}} \operatorname{sign}\left(\eta_{\delta_{1}}\right) \theta_{\delta_{1}} e_{\sigma\left(\delta_{1}\right)} .
$$

Note from the above argument that

$$
T\left(x_{1}\right)=\frac{1}{2^{1 / \beta}} \theta_{\delta_{0}} e_{\delta_{0}}-\frac{1}{2^{1 / \beta}} \operatorname{sign}\left(\eta_{\delta_{1}}\right) e_{\delta_{1}} .
$$

It follows that

$$
\begin{aligned}
1=\left\|x_{0}^{+}-x_{1}\right\| & \geq\left\|T\left(x_{0}^{+}\right)-T\left(x_{1}\right)\right\| \\
& \geq\left|\eta_{\delta_{0}}^{+}-\frac{1}{2^{1 / \beta}} \theta_{\delta_{0}}\right|^{\beta}+\left|\eta_{\delta_{1}}+\frac{1}{2^{1 / \beta}} \operatorname{sign}\left(\eta_{\delta_{1}}\right)\right|^{\beta} \\
& >\left|\eta_{\delta_{0}}^{+}-\frac{1}{2^{1 / \beta}} \theta_{\delta_{0}}\right|^{\beta}+\frac{1}{2} .
\end{aligned}
$$

Thus $\operatorname{sign}\left(\eta_{\delta_{0}}^{+}\right)=\theta_{\delta_{0}}$. Similarly, we can also obtain $\operatorname{sign}\left(\eta_{\delta_{0}}^{-}\right)=-\theta_{\delta_{0}}$.

By (2.4), we have $\left|\eta_{\delta_{0}}^{ \pm}\right| \geq(1 / 2)^{1 / \beta}$ and observe that

$$
\begin{aligned}
2^{\beta-1}=\left\|x_{0}^{+}-x_{0}^{-}\right\| & \geq\left\|T\left(x_{0}^{+}\right)-T\left(x_{0}^{-}\right)\right\| \\
& \geq\left|\eta_{\delta_{0}}^{+}-\eta_{\delta_{0}}^{-}\right|^{\beta}=\left(\left|\eta_{\delta_{0}}^{+}\right|+\left|\eta_{\delta_{0}}^{-}\right|\right)^{\beta} .
\end{aligned}
$$

Consequently,

$$
\eta_{\delta_{0}}^{+}=\frac{1}{2^{1 / \beta}} \theta_{\delta_{0}} \quad \text { and } \quad \eta_{\delta_{0}}^{-}=-\frac{1}{2^{1 / \beta}} \theta_{\delta_{0}} .
$$

Moreover, this and the inequality becoming an equality in (2.7) imply that

$$
T\left(x_{0}^{+}\right)(\delta)=T\left(x_{0}^{-}\right)(\delta) \quad \forall \delta \neq \delta_{0} .
$$


Now using the same technique as in Lemma 2.3, we define

$$
\phi(\lambda)=\left\|T(f(\lambda))-T\left(x_{0}^{+}\right)\right\|-\left\|T(f(\lambda))-T\left(x_{0}^{-}\right)\right\|
$$

for all $\lambda \in[0,1]$ where $f(\lambda)=\left((1-\lambda) x_{0}^{+}+\lambda x_{0}^{-}\right) /\left(\left\|(1-\lambda) x_{0}^{+}+\lambda x_{0}^{-}\right\|^{1 / \beta}\right)$.

Since $\phi$ is continuous on $[0,1]$ and $\phi(0) \phi(1)<0$, there is a $\lambda_{0} \in(0,1)$ such that

$$
\left\|T\left(f\left(\lambda_{0}\right)\right)-T\left(x_{0}^{+}\right)\right\|=\left\|T\left(f\left(\lambda_{0}\right)\right)-T\left(x_{0}^{-}\right)\right\| .
$$

Hence by the form of $T\left(x_{0}^{ \pm}\right)$given by (2.8) and (2.9) we see that

$$
T\left(f\left(\lambda_{0}\right)\right)\left(\delta_{0}\right)=0 .
$$

So

$$
\left\|f\left(\lambda_{0}\right)-e_{\sigma\left(\delta_{0}\right)}\right\| \geq\left\|T\left(f\left(\lambda_{0}\right)\right)-T\left(e_{\sigma\left(\delta_{0}\right)}\right)\right\|=2
$$

yields $f\left(\lambda_{0}\right)\left(\sigma\left(\delta_{0}\right)\right)=0$.

It follows that $f\left(\lambda_{0}\right)=e_{\gamma_{0}}$, that is, $T\left(e_{\gamma_{0}}\right)\left(\delta_{0}\right)=0$. This contradicts the choice of $\delta_{0}$. Thus the proof is complete.

REMARK 2.5. In the case where $\operatorname{dim}\left(\ell^{\beta}(\Gamma)\right)<\infty$, that is, the cardinality of $\Gamma$ is finite, the above conclusion that $T$ is an isometry cannot be simply obtained by a compactness argument or Freudenthal and Hurewicz's result [9] which states that every nonexpansive map from a totally bounded metric space onto itself must be an isometry since the nonexpansive map is not assumed to be from $S\left(\ell^{\beta}(\Gamma)\right)$ onto itself. The statement of Theorem 2.4 remains valid if we consider the quasi-Banach space consisting of the all the points $x=\left\{\xi_{\gamma}\right\}_{\gamma \in \Gamma} \in \ell^{\beta}(\Gamma)$ with the quasi-norm $\|x\|_{\beta}=$ $\left(\sum\left|\xi_{\gamma}\right|^{\beta}\right)^{1 / \beta}$ for $0<\beta<1$.

COROLLARY 2.6. Every surjective nonexpansive mapping $T: S\left(\ell^{\beta}(\Gamma)\right) \rightarrow S\left(\ell^{\beta}(\Delta)\right)$ can be extended to a linear surjective isometry on $\ell^{\beta}(\Gamma)$.

REMARK 2.7. We can see from Lemma 2.3 that the surjection assumption of $T$ in Theorem 2.4 and Corollary 2.6 in fact can reduce to $\left\{ \pm e_{\delta}\right\}_{\delta \in \Delta} \subset T\left(S\left(\ell^{\beta}(\Gamma)\right)\right)$. On the other hand, by Theorem 2.4, we have in fact shown that every nonexpansive map $T$ from $S\left(\ell^{\beta}(\Gamma)\right)$ onto $S\left(\ell^{\beta}(\Delta)\right)$ ensures that for every $\gamma \in \Gamma$, supp $T\left(e_{\gamma}\right)$ is a singleton. However, without the assumption of surjectivity or $\left\{ \pm e_{\delta}\right\}_{\delta \in \Delta} \subset T\left(S\left(\ell^{\beta}(\Gamma)\right)\right)$ this is not always true. For example, let $T: S\left(\ell_{(2)}^{\beta}\right) \rightarrow S\left(\ell_{(3)}^{\beta}\right)$ be defined by

$$
T\left(\xi_{1} e_{1}+\xi_{2} e_{2}\right)=\xi_{1}\left(1 / 2^{1 / \beta} e_{1}+1 / 2^{1 / \beta} e_{2}\right)+\xi_{2} e_{3}
$$

where $\left\{\xi_{1}, \xi_{2}\right\} \subset \mathbb{R}$ satisfies $\left|\xi_{1}\right|^{\beta}+\left|\xi_{2}\right|^{\beta}=1$. Then $T$ is an isometry, but $e_{1}, e_{2} \notin$ $T\left(S\left(\ell_{(2)}^{\beta}\right)\right)$ and supp $T\left(e_{1}\right)=\{1,2\}$. Considering this example, we give a more general result for expansive maps on $S\left(\ell^{\beta}(\Gamma)\right)$. 
THEOREM 2.8. Let $T$ be an expansive map from $S\left(\ell^{\beta}(\Gamma)\right)$ to $S\left(\ell^{\beta}(\Delta)\right)$ such that $T\left(S\left(\ell^{\beta}(\Gamma)\right)\right)=S(F)$, where $F$ is a linear closed subspace of $\ell^{\beta}(\Delta)$. Then $T$ is an isometry and can be extended to a linear isometry on $\ell^{\beta}(\Gamma)$.

Proof. Since $\|T(x)-T(y)\| \geq\|x-y\|$ for all $x, y \in S\left(\ell^{\beta}(\Gamma)\right)$, we see that $T$ is injective and its inverse $T^{-1}$ is nonexpansive. Note that $T^{-1}\left(T\left(e_{\gamma}\right)\right)=e_{\gamma}$ and $T^{-1}\left(T\left(-e_{\gamma}\right)\right)=-e_{\gamma}$ holds for all $\gamma \in \Gamma$. By the same argument as in Lemma 2.3, we deduce that

$$
T\left(-e_{\gamma}\right)=-T\left(e_{\gamma}\right)
$$

It follows that, for every $\gamma_{1} \neq \gamma_{2}$,

$$
\left\|T\left(e_{\gamma_{1}}\right)+T\left(e_{\gamma_{2}}\right)\right\| \geq\left\|e_{\gamma_{1}}+e_{\gamma_{2}}\right\|=2 .
$$

Hence

$$
\left\|T\left(e_{\gamma_{1}}\right)+T\left(e_{\gamma_{2}}\right)\right\|=\left\|T\left(e_{\gamma_{1}}\right)-T\left(e_{\gamma_{2}}\right)\right\|=2,
$$

which together with Lemma 2.1 guarantees that

$$
\operatorname{supp} T\left(e_{\gamma_{1}}\right) \cap \operatorname{supp} T\left(e_{\gamma_{2}}\right)=\emptyset .
$$

Thus $y=\sum \xi_{\gamma} T\left(e_{\gamma}\right)$ has norm one for every $\sum \xi_{\gamma} e_{\gamma} \in S\left(\ell^{\beta}(\Gamma)\right)$. Since $F$ is a linear closed subspace and $T\left(S\left(\ell^{\beta}(\Gamma)\right)\right)=S(F)$, it follows that $y \in T\left(S\left(\ell^{\beta}(\Gamma)\right)\right)$. Hence there is an element $x=\sum \alpha_{\gamma} e_{\gamma} \in S\left(\ell^{\beta}(\Gamma)\right)$ such that $T(x)=y$.

For any $\xi_{\gamma} \neq 0$, by (2.10) and (2.11) we get

$$
\left\|T(x)-\operatorname{sign}\left(\xi_{\gamma}\right) T\left(e_{\gamma}\right)\right\|=\left(1-\left|\xi_{\gamma}\right|\right)^{\beta}+1-\left|\xi_{\gamma}\right|^{\beta} .
$$

Furthermore,

$$
\left\|x-\operatorname{sign}\left(\xi_{\gamma}\right) e_{\gamma}\right\|=\left|\operatorname{sign}\left(\xi_{\gamma}\right)-\alpha_{\gamma}\right|^{\beta}+1-\left|\alpha_{\gamma}\right|^{\beta} .
$$

Thus by the fact that $T$ is expansive,

$$
\left|\operatorname{sign}\left(\xi_{\gamma}\right)-\alpha_{\gamma}\right|^{\beta}-\left|\alpha_{\gamma}\right|^{\beta} \leq\left(1-\left|\xi_{\gamma}\right|\right)^{\beta}-\left|\xi_{\gamma}\right|^{\beta} .
$$

It follows that $\left|\alpha_{\gamma}\right| \geq\left|\xi_{\gamma}\right|$. This yields $1=\sum\left|\alpha_{\gamma}\right|^{\beta} \geq \sum\left|\xi_{\gamma}\right|^{\beta}=1$, which combined with (2.12) ensures that for every $\gamma, \alpha_{\gamma}=\xi_{\gamma}$ even if $\xi_{\gamma}=0$. That is,

$$
T\left(\sum \xi_{\gamma} e_{\gamma}\right)=\sum \xi_{\gamma} T\left(e_{\gamma}\right)
$$

for every $\sum \xi_{\gamma} e_{\gamma} \in S\left(\ell^{\beta}(\Gamma)\right)$.

Finally, by its property given by (2.13), $T$ is clearly an isometry and the desired extension $\widetilde{T}$ defined by

$$
\widetilde{T}\left(\sum \bar{\xi}_{\gamma} e_{\gamma}\right)=\sum \bar{\xi}_{\gamma} T\left(e_{\gamma}\right) \quad \forall \sum \bar{\xi}_{\gamma} e_{\gamma} \in \ell^{\beta}(\Gamma)
$$

It is plain that $\widetilde{T}$ is a linear isometry on $\ell^{\beta}(\Gamma)$ and its restriction to $S\left(\ell^{\beta}(\Gamma)\right)$ is just $T$. The proof is complete. 
REMARK 2.9. If $\beta=1$, then some minor modifications of the previous example give a counterexample showing that there is an expansive map or, to be precise, an isometry between $S\left(\ell^{1}(\Gamma)\right)$ and $S\left(\ell^{1}(\Delta)\right)$ which cannot be linearly extended to the whole space. In fact, let $T: S\left(\ell_{(2)}^{1}\right) \rightarrow S\left(\ell_{(3)}^{1}\right)$ be defined by

$$
T\left(\xi_{1} e_{1}+\xi_{2} e_{2}\right)= \begin{cases}\xi_{1}\left(1 / 4 e_{1}+3 / 4 e_{2}\right)+\xi_{2} e_{3} & \text { if } \xi_{1} \geq 0 \\ \xi_{1}\left(1 / 2 e_{1}+1 / 2 e_{2}\right)+\xi_{2} e_{3} & \text { otherwise }\end{cases}
$$

where $\left\{\xi_{1}, \xi_{2}\right\} \subset \mathbb{R}$ satisfies $\left|\xi_{1}\right|+\left|\xi_{2}\right|=1$. It is easy to check that $T$ does not satisfy the condition of Theorem 2.8 since $-T\left(S\left(\ell_{(2)}^{1}\right)\right) \nsubseteq T\left(S\left(\ell_{(2)}^{1}\right)\right)$, and that $T$ is an isometry which cannot be linearly extended to $\ell_{(2)}^{1}$ because it is not even an odd operator.

\section{Acknowledgements}

I express my sincere gratitude to Professor Ding for his support and encouragement and to the referee for the valuable comments and suggestions.

\section{References}

[1] G. M. An, 'Isometries on unit sphere of $\left(\ell^{\beta_{n}}\right)$ ', J. Math. Anal. Appl. 301 (2005), $249-254$.

[2] G. G. Ding, 'The 1-Lipschitz mapping between the unit spheres of two Hilbert spaces can be extended to a real linear linear isometry of the whole space', Sci. China Ser. A 45(4) (2002), 479-483.

[3] G. G. Ding, 'The isometric extension problem in the unit spheres of $\ell^{p}(\Gamma)(p>1)$ type spaces', Sci. China Ser. A 46(3) (2003), 333-338.

[4] G. G. Ding, 'On the extension of isometries between unit spheres of $E$ and $C(\Omega)$ ', Acta Math. Sin. (Engl. Ser.) 19(4) (2003), 793-800.

[5] G. G. Ding, 'The representation theorem of onto isometric mappings between two unit spheres of $\ell^{1}(\Gamma)$-type spaces and the application to the isometric extension problem', Acta Math. Sin. (Engl. Ser.) 20(6) (2004), 1089-1094.

[6] G. G. Ding, 'The representation of onto isometric mappings between two spheres of $\ell^{\infty}$-type spaces and the application on isometric extension problem', Sci. China Ser. A 34(2) (2004), 157-164 (in Chinese); 47(5) (2004), 722-729 (in English).

[7] G. G. Ding, 'The isometric extension of the into mapping from a $\mathcal{L}^{\infty}(\Gamma)$-type space to some Banach space', Illinois J. Math. 51(2) (2007), 445-453.

[8] X. N. Fang, 'On extension of 1-Lipschitz mapping between two unit spheres of $\ell^{p}(\Gamma)$-type spaces $(1<p<\infty)$ ', J. Math. Res. Exposition 29(4) (2009), 687-692.

[9] H. Freudenthal and W. Hurewicz, 'Dehnungen, Verkurzungen, Isometrien', Fund. Math. 26 (1936), 120-122.

[10] R. Liu, 'On extension of isometries between unit spheres of $\mathcal{L}^{\infty}(\Gamma)$-type space and a Banach space E', J. Math. Anal. Appl. 333 (2007), 959-970.

[11] D. N. Tan, 'Nonexpansive mappings on the unit spheres of some Banach spaces', Bull. Aust. Math. Soc. 80(1) (2009), 139-146.

[12] D. Tingley, 'Isometries of the unit sphere', Geom. Dedicata 22 (1987), 371-378.

[13] J. Wang, 'On extension of isometries between unit spheres of $A L_{p}$-spaces $(0<p<\infty)$ ', Proc. Amer. Math. Soc. 132(10) (2004), 2899-2909. 
[14] R. D. Wang, 'On extension of 1-Lipschitz mappings between $\ell^{1}(\Gamma)$-type spaces', Acta Sci. Natur. Univ. Nankai, to appear.

[15] X. Z. Yang, 'On extension of isometries between unit spheres of $L_{p}(\mu)$ and $L_{p}(\nu, H)(1<p \neq$ 2, $H$ is a Hilbert space)', J. Math. Anal. Appl. 323 (2006), 985-992.

DONG-NI TAN, School of Mathematical Science and LPMC, Nankai University, Tianjin 300071, PR China

e-mail:0110127@mail.nankai.edu.cn 